\title{
Localization of Low Energy Losses and the Mixed Dynamic Form Factor
}

\author{
P. Schattschneider*, C. Hébert*, B. Jouffrey** \\ *Institut für Festkörperphysik, TU Wien, Wiedner Hauptstraße 8-10 1040 Vienna, Austria \\ ** LMSS-Mat, École Centrale Paris, Grande Voie des Vignes, 92295, Châtenay-Malabry, France
}

The mixed dynamic form factor (MDFF) $S\left(\mathbf{q}, \mathbf{q}^{\prime}, E\right)$ of a target is a density matrix in reciprocal space q. As such, it contains information on the density (on the main diagonal) and on the mutual coherence (on the skew diagonal). The Fourier transform of the MDFF with respect to $\left(\mathbf{q},-\mathbf{q}^{\prime}\right)$ (denoted $r$ $\mathrm{MDFF}$ ), relates to an energy-dependent density matrix in real space

$$
S\left(\mathbf{r}, \mathbf{r}^{\prime}, E\right):=F T_{\mathbf{q},-\mathbf{q}^{\prime}}\left[S\left(\mathbf{q}, \mathbf{q}^{\prime}, E\right)\right]=\int_{-E}^{0} \rho_{E_{i}}\left(\mathbf{r}, \mathbf{r}^{\prime}\right) \rho_{E_{i}+E}\left(\mathbf{r}^{\prime}, \mathbf{r}\right) d E_{i}
$$

where $\rho_{E_{i}}\left(\mathbf{r}, \mathbf{r}^{\prime}\right)$ is the density matrix of the target for states of energy $E_{i}$. In other words, the Fourier transform of the MDFF is an energy correlation function between the density matrices of occupied (initial) states and unoccupied (final) states, with energy difference $E$ between these states. $E$ equals the energy loss of the probe electron. The overlap of initial and final electron density matrices determines the outcome of inelastic scattering experiments.

The inelastic scattering cross section for ionization by two coherently incident plane electron waves with wave vectors $\mathbf{k}_{1}$ and $\mathbf{k}_{2}$ is the sum of two direct terms and an interference term that is proportional to the MDFF [1]. It resembles the intensity oscillations of a conventional interferometer. The proportionality factor depends on the orientation of the primary electron beam relative to the crystal lattice, on the position of the scattering event, and on the thickness of the specimen. Hence the interference term can be tuned by setting up channeling or antichanneling conditions.

This is the basis for ALCHEMI and ELCE and has recently been applied to interpret faint changes in ELNES in terms of transitions to different final states [2]. Integrating out one variable in the MDFF we find that the Fourier transform of the density in real space is the projection of the MDFF onto the skew diagonal in reciprocal space:

$$
F T_{\mathbf{r}}[S(\mathbf{r}, \mathbf{r}, E)]=\int S\left(\mathbf{q}^{\prime}, \mathbf{q}+\mathbf{q}^{\prime}, E\right) d^{3} q^{\prime}
$$

A high value of the MDFF on the skew diagonal indicates strong localization of the corresponding excitation. Therefore, it should be possible to analyze eigenmodes in the energy loss spectrum with respect to their localization.

In particular, the MDFF of a delocalized collective mode should vanish, and the energy loss spectrum should be independent of the channeling conditions. Low loss spectra of $\mathrm{Si}$ and $\mathrm{TiO}_{2}$ rutile taken in diffraction show differences between channeling and antichanneling spectra. The variations in the Si L edge - see Fig. 1 - were interpreted as channeling enhanced monopole transitions. This can be related to localization since $\mathrm{p} \rightarrow \mathrm{p}$ transitions are more localized than $\mathrm{p} \rightarrow \mathrm{s}$ transitions. The same holds for the $3 \mathrm{p}$ semicore transition in rutile at $\approx 39 \mathrm{eV}$ where time-dependent density functional theory (TD-DFT) 
in the local density approximation showed strong localization [3].

More surprising is the fact that the main peak at $\approx 25 \mathrm{eV}$ changes shape - see Fig. 2 . This is clear evidence that the collective mode in rutile is somewhat localized. This was recently confirmed by a TD-DFT calculation of the plasmon dispersion [3]. Local field effects change the plasma frequency dramatically for non-vanishing $\mathrm{q}$. The effect is most prominent between 10 and $14 \mathrm{eV}$, with an increase and subsequent decrease in cross section. In this loss region a significant structure appears in the experimental difference spectrum. How this feature exactly relates to the off-diagonal elements of the dielectric matrix remains to be seen. [4]

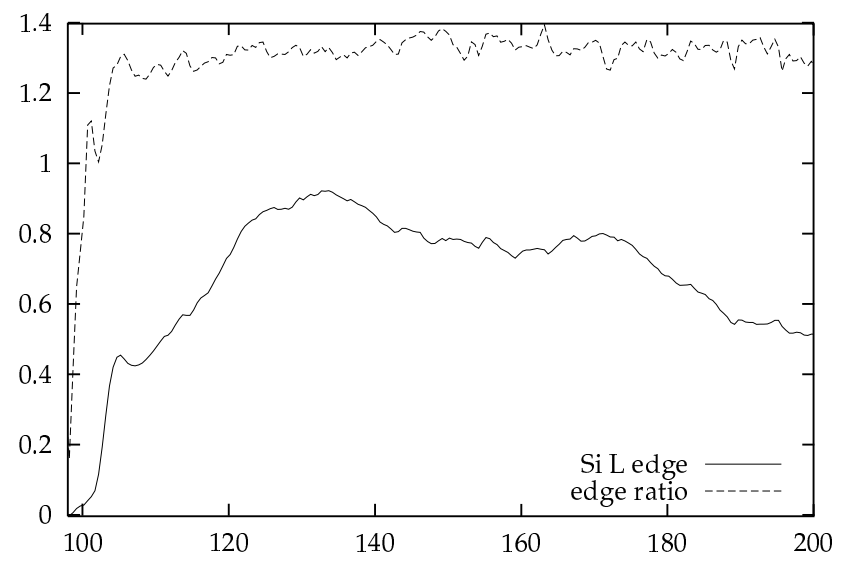

Figure 1: Si L edge and ratio of spectra under channeling to antichanneling conditions. Localization is strong at the edge onset.

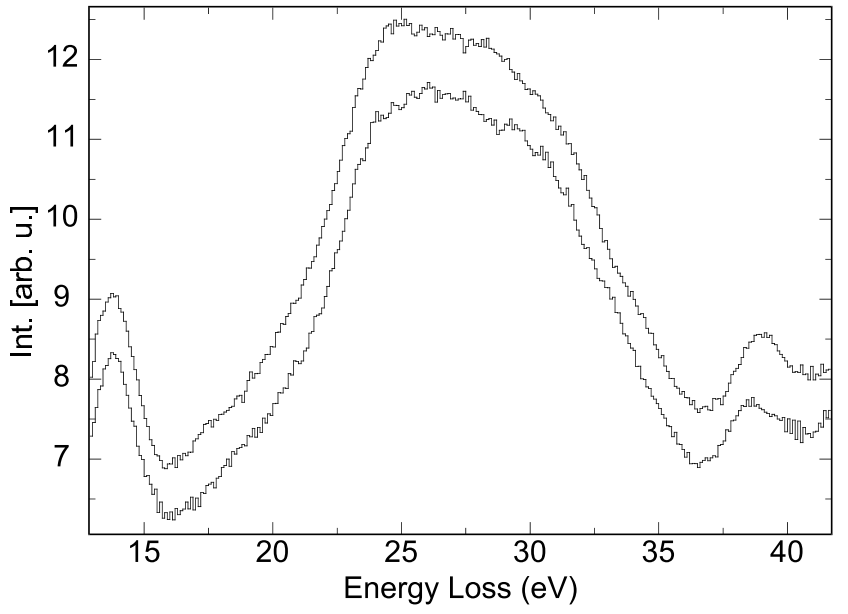

Figure 2: Low loss spectrum of rutile under channeling (upper curve) and antichanneling conditions (curve below).

\section{References}

[1] P. Schattschneider et al. Ultramicrosc. 86 (2000) 343.

[2] M. Nelhiebel et al. Phys. Rev. Lett. 85 (2000) 1847.

[3] N. Vast et al., Phys. Rev. Lett. 88 (2001) 037601-7.

[4] This work was sponsored by a joint grant of the CNRS and the Vienna University of Technology (PICS 913). 